\title{
FATOU THEOREMS FOR EIGENFUNCTIONS OF THE INVARIANT DIFFERENTIAL OPERATORS ON SYMMETRIC SPACES
}

\author{
BY
}

\section{H. LEE MICHELSON( $\left.{ }^{1}\right)$}

\begin{abstract}
On a Riemannian symmetric space of noncompact type we introduce a generalization of the Poisson kernel which may be used to generate simultaneous eigenfunctions of the invariant differential operators with eigenvalues not necessarily zero. We investigate the boundary behavior of . our generalized Poisson integrals, extending to them many of the Fatou-type theorems known for harmonic functions.
\end{abstract}

Introduction. A classical theorem of Fatou states that a bounded measurable function on the circle is equal to the nontangential limit of its Poisson integral almost everywhere. Korányi in [12] and [13] (cf. also [11]) has defined several types of admissible convergence of a function on a Riemannian symmetric space of noncompact type to a function on any Furstenberg-Satake boundary of that symmetric space and has proven the analogous theorem for semirestricted admissible convergence, extending a result of Helgason and himself [8] for radial convergence. In the case of a symmetric space of rank one, the result had also been proven in a more complicated manner by Knapp [10]; in this case the theorem extends easily to all integrable functions on the (in this case unique) boundary and even to finite regular signed measures on the boundary. (By pointwise convergence to a measure almost everywhere we understand pointwise convergence almost everywhere to its Radon-Nikodym derivative.) Some extension of the theorem for arbitrary noncompact symmetric spaces to function spaces on the boundary which are larger than $L^{\infty}$ has been carried out recently by Lindahl [14].

The Poisson kernel, whose integrals against functions (or, more generally, measures, distributions, etc.) on the maximal Furstenberg-Satake boundary generate

Received by the editors February 9, 1972.

AMS (MOS) subject classifications (1970). Primary 53C35, 43 A85.

Key words and phrases. Generalized Poisson integrals, unrestricted admissible convergence, products of rank-one spaces, restricted admissible convergence.

(1) Some of the results of this paper were included in the author's Ph. D. thesis at Yeshiva University, where the author was supported by a university assistantship. The author is now a C. L.E. Moore instructor at the Massachusetts Institute of Technology, partially supported by NSF grant P22930. The author wishes to express his gratitude to God and to Dr. A. Korányi of Yeshiva University and Dr. S. Helgason of the Massachusetts Institute of Technology for invaluable assistance at all stages of this work. 
harmonic functions on the symmetric space, is only one example of a type of exponential kernel which can be used to generate simultaneous eigenfunctions of the invariant differential operators on the symmetric space; namely, kernels of the form $e^{-(i \lambda+\rho)\left(H\left(g^{-1} k\right)\right)}$, where $H$ denotes the projection on the abelian part of the Iwasawa decomposition, $\rho$ denotes the half-sum of the positive restricted roots, and $\lambda$ is any complex linear function on the Cartan subspace a (precise definitions to be given in $\$ 1$ ). (The Poisson kernel corresponds to the case $\lambda=-i \rho$. ) Karpelevič [9] (as, in a less precise way, also Furstenberg [2]) has proven that every nonnegative eigenfunction of all invariant differential operators is the integral with respect to some finite positive regular measure on the maximal boundary of such an exponential kernel with $\lambda \epsilon-i \bar{a}_{+}^{*}$, where $\bar{a}_{+}^{*}$ is the closed positive Weyl chamber. ( $\lambda$ determines the eigenvalues.) In the rank-one case Helgason [6] has proven that every eigenfunction of all invariant differential operators is the integral of such an exponential kernel with $\lambda \epsilon a^{*}-i \bar{a}_{+}^{*}$ with respect to some "functional" on the boundary, although he has characterized the boundary functionals which occur only in the case of the noneuclidean disc. (In that case they are the analytic functionals.)

One is prompted to study the boundary behavior of the eigenfunctions of the invariant differential operators obtained from these more general integrals. Since the integral gotten from the function constant 1 on the boundary is some spherical function, which we denote by $\phi_{\lambda}$, it is natural to divide the eigenfunction by $\phi_{\lambda}$ and to ask whether this ratio converges in some sense to its boundary values. This question was first asked by Helgason. In [7] he states that in the case of the noneuclidean disc the Fourier coefficients of the above-mentioned ratio on any circle centered at the origin approach the Fourier coefficients of the boundary analytic functional as the noneuclidean radius of the circle approaches infinity, where $\lambda=0$ (in Helgason's notation, $\mu=1 / 2$ ) or $\lambda \epsilon a^{*}-i a_{+}^{*}$ (in his notation, $\operatorname{Re} \mu>1 / 2$ ). Of course, a stronger form of convergence is desirable, at least for the integrals of elements of some "nice" function spaces on the boundary. Helgason, in an unpublished manuscript, does prove radial convergence of the ratio under consideration to its boundary value at a point of continuity of a bounded measurable function on the maximal boundary of any noncompact symmetric space for $\lambda \in a^{*}-i a_{+}^{*}$ (in the rank-one case, also for $\lambda=0$ ). The proof is contained essentially in that of Lemmas 1.2 and 1.3 of Chapter IV of [6].

In this paper we shall extend the above result of Helgason for radial convergence at points of continuity to unrestricted admissible convergence at Lebesgue points (in particular, almost everywhere). In the case of spaces of rank one and their products, and for some values of $\lambda$ even in the general case, 
we shall also extend Helgason's result for $L^{\infty}$ to larger function spaces.

In $\$ 1$ we define the generalized Poisson kernel, develop some of its propefties, and use them to prove some "soft" Fatou-type theorems (for continuous functions, and for convergence in function-space topologies). In $\$ 2$ we establish those results for pointwise unrestricted admissible convergence almost everywhere which are known for arbitrary symmetric spaces of noncompact type. In $\$ 3$ we discuss the additional results known for products of spaces of rank one.

1. Generalized Poisson integrals. Let $X$ be a Riemannian symmetric space of noncompact type; $G$, the connected isometry group of $X ; K$, the isotropy group of the point $o \in X$. Letting $g$, denote the Lie algebras of $G$ and $K$, respectively, we can form a Cartan decomposition of $g: g=t+h$. Let $a$ be a maximal abelian subspace of $p$. Introduce an ordering in $a^{*}$, the dual of $a$, and let $n$ and $\bar{n}$, respectively, denote the sums of the eigenspaces of $\operatorname{ad}_{g}(a)$ corresponding to the positive and to the negative eigenvalues. We can form the Iwasawa decompositions $g=\mathfrak{E}+a+n, G=K A N$, where $A$ and $N$ are the analytic subgroups of $G$ corresponding to the subalgebras $a$ and $n$, respectively, of $g$. For $g \in G$, we shall write $k(g), H(g), n(g)$ to denote the elements of $K$, $a$, and $N$, respectively, such that $g=k(g) \exp H(g) n(g)$. If $M$ is the centralizer of $a$ in $K$, then $K / M \cong G / M A N$ is the maximal Furstenberg-Satake boundary of $X$. There is a unique $K$-invariant probability measure on $K / M$; we denote it by $d k_{M}$. We denote the set of positive eigenvalues of $\operatorname{ad}_{\mathfrak{g}}(a)$ by $Q^{+}$and their half-sum (with multiplicities) by $\rho$. We let $P^{+}=\left\{a \in Q^{+} \mid 1 / 2 \alpha \notin Q^{+}\right\}$. We let $a_{+}^{*}=\left\{\mu \in a^{*} \mid\right.$ $\left.\left(\forall \alpha \in P^{+}\right)\langle a, \mu\rangle>0\right\}$, where $\langle$,$\rangle is the bilinear form on a^{*}$ dual to the Killing form of $g$ on $a$. (We also write $a_{+}$for $\left\{H \in a \mid\left(\forall \alpha \in P^{+}\right) \alpha(H)>0\right\}$.)

For $\lambda \in a_{\mathbf{C}}^{*}$, the complexification of $a^{*}$, we write

$$
\begin{gathered}
\phi_{\lambda}(g \cdot o)=\int_{K / M} e^{-(i \lambda+\rho)\left(H\left(g^{-1} k\right)\right)} d k_{M}, \\
P_{\lambda}(g \cdot o, k M)=\left[\phi_{\lambda}(g \cdot o)\right]^{-1} e^{-(i \lambda+\rho)\left(H\left(g^{-1} k\right)\right)}
\end{gathered}
$$

wherever $\phi_{\lambda}(g \cdot o) \neq 0$. It is clear that $\phi_{\lambda}$ and $P_{\lambda}$ are well-defined functions on $X$ and $X_{\lambda} \times K / M$, respectively, where $X_{\lambda}=\left\{x \in X \mid \phi_{\lambda}(x) \neq 0\right\}$. If $f$ is an integrable function on $K / M$, we shall write

$$
F_{\lambda}(x)=\int_{K / M} P_{\lambda}(x, k M) f(k M) d k_{M},
$$

for $x \in X_{\lambda}$. Sometimes we shall, abusing the notation slightly, indicate a measure, or even a distribution, on $K / M$ also by $f(k M) d k_{M}$, in which case the integral of $P_{\lambda}(x, k M)$ with respect to $f(k M) d k_{M}$ will still be denoted by $F_{\lambda}(x)$.

For $\lambda \in a^{*}-i a_{+}^{*}$, Harish-Chandra [4] has derived an asymptotic formula for $\phi_{\lambda}$, as follows: 


$$
\phi_{\lambda}(k a \cdot o) \sim c(\lambda) e^{(i \lambda-\rho)(\log a)} \quad \text { as } a \rightarrow+\infty,
$$

provided that $|c(\lambda)|<\infty$, where $c$ is some meromorphic function on $a_{C}^{*}$. (Here and in the sequel $k \in K, a \in A, \log$ denotes the inverse of $\left.\exp _{g}\right|_{a}$, and the notation $a \rightarrow+\infty$ means $\left(\forall \alpha \in P^{+}\right) \alpha(\log a) \rightarrow+\infty$. Note that $\phi_{\lambda}$ is $K$-invariant.) The function $c(\lambda)$ has been computed by Gindikin and Karpelevic [3] and is given by the formula

$$
\begin{aligned}
c(\lambda) & =\int_{\bar{N}} e^{-(i \lambda+\rho)(H(\bar{n}))} d \bar{n} \\
& =c_{0} \prod_{a \in P^{+}} \frac{\Gamma\left(\left\langle i \lambda, \alpha_{0}\right\rangle\right) 2^{-\left\langle i \lambda, a_{0}\right\rangle}}{\Gamma\left(1 / 2\left(1 / 2 m_{a}+1+\left\langle i \lambda, \alpha_{0}\right\rangle\right)\right) \Gamma\left(1 / 2\left(1 / 2 m_{a}+m_{2 a}+\left\langle i \lambda, \alpha_{0}\right\rangle\right)\right.},
\end{aligned}
$$

where $\bar{N}$ is the analytic subgroup of $G$ corresponding to $\bar{n}, c_{0}$ is some constant, $\alpha_{0}=\alpha /\langle\alpha, \alpha\rangle$, and $m_{a}$ denotes the multiplicity of $\alpha$. Since $\left.\langle i \lambda, \alpha\rangle\right\rangle 0$, we have in fact that $0<|c(\lambda)|<\infty$. The integral in (1.2) is absolutely convergent.

In the case rank $X \equiv \operatorname{dim} a=1$, Harish-Chandra [4, p. 302] gives an explicit formula for $\phi_{\lambda}$ in terms of the hypergeometric function $F$ :

$$
\begin{aligned}
\phi_{\lambda}(k a \cdot o)=F\left(1 / 4\left(m_{\alpha}+2 m_{2 a}-2 i \lambda\left(H_{0}\right)\right), 1 / 4\left(m_{\alpha}+2 m_{2 a}+2 i \lambda\left(H_{0}\right)\right) ;\right. \\
\left.1 / 2\left(m_{a}+m_{2 a}+1\right) ;-(\sinh \alpha(\log a))^{2}\right)
\end{aligned}
$$

where $a$ is the unique element of $P^{+}, H_{0} \in a$ such that $\alpha\left(H_{0}\right)=1$. This formula permits us to determine the asymptotic behavior of $\phi_{\lambda}$ as $a \rightarrow+\infty$ also for $\lambda=0$. Namely, an application of formula (18) or (19) on p. 63 of [1] yields

$$
\phi_{0}(k a \cdot o) \sim \frac{\Gamma\left(1 / 2\left(m_{a}+m_{2 a}+1\right)\right) 2^{1 / 2\left(m_{a}+m_{2 a}\right)}}{\Gamma\left(1 / 4\left(m_{a}+2 m_{2 a}\right)\right) \Gamma\left(1 / 4\left(m_{a}+2\right)\right)} a(\log a) e^{-\rho(\log a)} .
$$

By substituting formula $(1.1)$ or (1.3) into the definition of $P_{\lambda}$, we obtain (1.4) $\quad P_{\lambda}(a \cdot o, k M) \sim\left\{\begin{array}{l}C_{\lambda} e^{(\rho-i \lambda)(\log a)} e^{-(i \lambda+\rho)\left(H\left(a^{-1} k\right)\right)}, \quad \lambda \in a^{*}-i a_{+}^{*}, \\ C_{0}[\alpha(\log a)]^{-1} e^{\rho(\log a)} e^{-\rho\left(H\left(a^{-1} k\right)\right)}, \quad \lambda=0, \text { rank } X=1,\end{array}\right.$ as $a \rightarrow+\infty$, where $C_{\lambda}$ is a nonzero constant depending on $\lambda$. (In particular, $a . o \in X_{\lambda}$ whenever $\alpha(\log a)$ is big enough for each $a \in P^{+}$.)

Now we shall prove a lemma which will enable us to replace $o$ in (1.4) by an arbitrary $x \in X$.

1.1 Lemma. If we write $a \cdot x=k^{\prime} a^{\prime} \cdot o, k^{\prime} \in K, a^{\prime} \in \exp \bar{a}_{+}$, then as $a \rightarrow+\infty, k^{\prime} M \rightarrow e M$ and $a^{\prime} \rightarrow+\infty$.

Proof. By an Iwasawa decomposition we may write $x=\bar{n}_{1} a \cdot o, \bar{n}_{1} \in \bar{N}$, $a_{1} \in A$. Then $a \cdot x=a \bar{n}_{1} a_{1} \cdot o=\bar{n}_{1}^{a} a a_{1} \cdot o$. (Here and in the sequel we use the notation $g^{b}=b g b^{-1}$.) Lemma 50 of [5] states that, for $\bar{n}_{0} \in \bar{N}, a_{0} \in A$, if 
$\bar{n}_{0} a_{0} \cdot o \in K a_{0}^{\prime} K, a_{0}^{\prime} \in \exp \bar{a}_{+}$, then $\lim _{a_{0 \rightarrow+\infty}}\left(\log a_{0}^{\prime}-\log a_{0}\right)=H\left(\bar{n}_{0}\right)$, and it follows from the proof (e.g., by applying Dini's theorem to the convergent net on the bottom of p. 604, which is obviously monotonically decreasing) that the convergence is uniform for $\bar{n}_{0}$ in a compact subset of $\bar{N}$. Now take $\bar{n}_{0}=\bar{n}_{1}^{a}, a_{0}=a a_{1}$. As $a \rightarrow+\infty, \bar{n}_{0} \rightarrow e$ and $a_{0} \rightarrow+\infty$. By uniformity (since $\bar{n}_{0}$ is eventually in a compact subset of $\bar{N}$ ), we may interchange limits and obtain that $a^{\prime} \rightarrow+\infty$ as $a \rightarrow+\infty$. Now, by compactness of $K / M, k^{\prime} M$ has an accumulation point as $a \rightarrow+\infty$. We shall show that the only possible accumulation point of $k^{\prime} M$ is $e M$, and hence that $k^{\prime} M \rightarrow e M$. Indeed, for $k_{0} M \neq e M$, $d\left(a \cdot x, k_{0} a^{\prime} \cdot o\right) \geq d\left(a \cdot o, k_{0} a^{\prime} \cdot o\right)-d(a \cdot x, a \cdot o)=d\left(a \cdot o, k_{0} a^{\prime} \cdot o\right)-d(x, o)$, which is eventually greater than some positive quantity as $a \rightarrow+\infty$ because $X$ has nonpositive curvature.

We are now prepared to prove the main lemma of this section.

1.2 Lemma. Let $\lambda \in a^{*}-i a_{+}^{*}$. The kernel $P_{\lambda}$ bas the following properties:

(i) For $x \in X_{\lambda}, k_{0} \in K ; k_{0} \cdot x \in X_{\lambda}$ and $P_{\lambda}\left(k_{0} \cdot x, k_{0} k M\right)=P_{\lambda}(x, k M)$.

(ii) For $x \in X_{\lambda}, \int_{K / M} P_{\lambda}(x, k M) d k_{M}=1$.

(iii) There exists a constant $M_{\lambda}$ such that, for each $x \in X$, as $a \rightarrow+\infty$ we have eventually $\int_{K / M}\left|P_{\lambda}(a \cdot x, k M)\right| d k_{M} \leq M_{\lambda}$.

(iv) For any neighborbood $U$ of $e M$ in $K / M, x \in X$,

$$
\lim _{a \rightarrow+\infty} \int_{K / M-U}\left|P_{\lambda}(a \cdot x, k M)\right| d k_{M}=0 .
$$

If rank $x=1$ these properties bold also for $\lambda=0$.

Proof. (i) and (ii) follow immediately from the definition of $P_{\lambda}$.

To prove (iii), we let $\eta \in a^{*}$ such that $\lambda \in a^{*}-i \eta$. Then

$$
\begin{aligned}
\left|P_{\lambda}(a \cdot x, k M)\right| & =\left|\phi_{\lambda}(a \cdot x)\right|^{-1} \phi_{-i \eta}(a \cdot x, k M) \\
& =\left|\phi_{\lambda}\left(a^{\prime} \cdot o\right)\right|^{-1} \phi_{-i \eta}\left(a^{\prime} \cdot o\right) P_{-i \eta}(a \cdot x, k M) \\
& \sim|c(\lambda)|^{-1} c(-i \eta) P_{-i \eta}(a \cdot x, k M) \text { as } a \rightarrow+\infty
\end{aligned}
$$

by (1.1) and Lemma 1.1 (where $a^{\prime}$ is as in Lemma 1.1). Thus by (ii) we may take $M_{\lambda}$ to be any number greater than $|c(\lambda)|^{-1} c(-i \eta)$.

By Lemma 1.1, (i), and the $K$-invariance of $d k_{M}$, it is enough to prove (iv) for $x=0$. To do so we transfer the integration from $K / M$ to $\bar{N}$. By a lemma of Bruhat and Harish-Chandra, the map $\bar{n} \rightarrow k(\bar{n}) M$ is a diffeomorphism from $\bar{N}$ onto an open dense subset of $K / M$. According to Lemma 44 of [4], this map has Jacobian equal to $e^{-2 \rho(H(\bar{n}))}$, if the Haar measure on $\bar{N}$ is normalized suitably. Let $V=\{\bar{n} \in \bar{N} \mid k(\bar{n}) \in U\}$. Then, for $\lambda \in a^{*}-i a_{+}^{*}$, by (1.4), 


$$
\begin{aligned}
& \lim _{a \rightarrow+\infty} \int_{K / M-U}\left|P_{\lambda}(a \cdot o, k M)\right| d k_{M}=\lim _{a \rightarrow+\infty} \int\left|P_{\lambda}(a \cdot o, k(\bar{n}) M)\right| e^{-2 \rho(H(\bar{n}))} d \bar{n} \\
& =\lim _{a \rightarrow+\infty}\left|C_{\lambda}\right| e^{(\rho-\eta)(\log a)} \int_{\bar{N}-V} e^{-(\rho+\eta)\left(H\left(a^{-1} k(\bar{n})\right)\right)} e^{-2 \rho(H(\bar{n}))} d \bar{n} \\
& =\lim _{a \rightarrow+\infty}\left|C_{\lambda}\right| e^{2 \rho(\log a)} \int_{\bar{N}-V} e^{-(\rho+\eta)\left(H\left(\bar{n}^{-1}\right)\right)} e^{(\eta-\rho)(H(\bar{n}))} d \bar{n}
\end{aligned}
$$

since clearly $H(g b)=H(g k(b))+H(b)$ and $H(g a)=H(g)+\log a$. Now we choose $\delta$ such that $0<\delta<1, \rho-\delta \eta \in a_{+}^{*}$. We have that

$$
\begin{aligned}
\lim _{a \rightarrow+\infty} \int_{K / M-U}\left|P_{\lambda}(a \cdot o, k M)\right| d k_{M} & \leq\left|C_{\lambda}\right| e^{2 \rho(\log a)} \int_{\bar{N}-V} e^{-(\rho+\delta \eta)\left(H\left(\bar{n}^{-a^{-1}}\right)\right)} d \bar{n} \\
& =\left|C_{\lambda}\right| \int_{\bar{N}-V^{a^{-1}}} e^{-(\rho+\delta \eta)(H(\bar{n}))} d \bar{n} .
\end{aligned}
$$

(We have made the change of variable $\bar{n} \rightarrow \bar{n}^{a}$, which has Jacobian $e^{-2 \rho(\log a)}$ (cf. [12, Theorem 2.6]).)

Since $V^{a^{-1}} \rightarrow \bar{N}$ as $a \rightarrow+\infty$, and $e^{-(\rho+\delta \eta)(H(\bar{n}))}$ is integrable by the absolute integrability in (1.2), we have $\lim _{a \rightarrow+\infty} \int_{K / M-U}\left|P_{\lambda}(a \cdot o, k M)\right| d k_{M}=0$.

For rank $X=1, \lambda=0$, an explicit formula of Helgason [7, Chapter III, Corollary 1.18 ] yields

$$
e^{-\rho\left(H\left(a^{-1} k(\bar{n})\right)\right)}
$$

$$
=e^{\rho(\log a)}\left[\frac{1+r|X|^{2}+4 r|Y|^{2}}{\left(1+r e^{2 a(\log a)}|X|^{2}\right)^{2}+4 r e^{4 a(\log a)}|Y|^{2}}\right]^{1 / 4\left(m_{a}+2 m_{2 a}\right)},
$$

where $\bar{n}=\exp X \exp Y, X \in g_{-\alpha}, Y \in g_{-2 a}$ (where $g_{\beta}$ denotes the $\beta$ eigenspace of $\left.\operatorname{ad}_{g}(a)\right),|Z|=B(Z, \theta Z)^{1 / 2}$ for $Z \in \bar{n}$ (where $B$ is the Killing form of $g$ and $\theta$ is the symmetry $), r=\left[4\left(m_{\alpha}+4 m_{2 a}\right)\right]^{-1}$. It follows from (1.4) that

$$
\begin{aligned}
P_{n}(a & \cdot o, k(\bar{n}) M) \\
\sim & C_{0}[a(\log a)]^{-1} e^{2 \rho(\log a)} \\
& \cdot\left[\frac{1+r|X|^{2}+4 r|Y|^{2}}{\left(1+r e^{2 a(\log a)}|X|^{2}\right)^{2}+4 r e^{4 \alpha(\log a)}|Y|^{2}}\right]^{1 / 4\left(m_{a}+2 m_{2 a}\right)}
\end{aligned}
$$

Since $2 \rho=\left(m_{a}+2 m_{2 a}\right) \alpha$,

$$
e^{2 \rho(\log a)}\left[\frac{\left(1+r|X|^{2}\right)^{2}+4|Y|^{2}}{\left(1+r e^{2 a(\log a)}|Y|^{2}\right)^{2}+4 r e^{4 a(\log a)}|Y|^{2}}\right]^{1 / 4\left(m_{a}+2 m_{2 a}\right)}
$$

remains bounded as $a \rightarrow+\infty$, uniformly for $\bar{n} \in \bar{N}-V$. Thus $P_{0}(a \cdot o, k(\bar{n}) M)$ approaches 0 as $a \rightarrow+\infty$, uniformly for $\bar{n} \in \bar{N}-V$. In particular, 


$$
\lim _{a \rightarrow+\infty} \int_{\bar{N}-V}\left|P_{0}(a \cdot o, k(\bar{n}) M)\right| e^{-2 \rho(H(\bar{n}))} d \bar{n}=0 .
$$

Since $X_{\lambda}$ is $K$-invariant (Lemma 1.2(i)), we can consider, for a function $F$ on $X_{\lambda}$ and a point $x \in X_{\lambda}$, the function $F^{x}$ on $K$ defined by $F^{x}(k)=F(k \cdot x)$. If $f(k M) d k_{M}$ is a distribution on $K / M$, we can define a distribution $\tilde{f}(k) d k$ on $K$ by

$$
\int_{K} \tilde{g}(k) \tilde{f}(k) d k=\int_{K / M}\left[\int_{M} \tilde{g}(k m) d m\right] f(k M) d k_{M},
$$

for $\tilde{g}$ any test function on $K$. It is cleat that if $f$ is a measure (respectively: an $L^{p}$ function, a continuous function) so is $\tilde{f}$. Using this notation we can now state a "soft" Fatou-type theorem:

1.3 Theorem. Let $\lambda \in a^{*}-i a_{+}^{*}$ or rank $X=1, \lambda=0$. For any $x \in X$, $\lim _{a \rightarrow+\infty} F^{a \cdot x}=\tilde{f}$

(i) uniformly, $f \in C(K / M)$,

(ii) in $L^{p}(K), f \in L^{p}(K / M), 1 \leq p<\infty$,

(iii) weak* against $L^{1}(K), f \in L^{\infty}(K / M)$,

(iv) weak* against $C(K), f$ a finite regular signed measure on $K / M$,

(v) weak* against $C^{\infty}(K), f$ a distribution on $K / M$.

Proof. We omit the details of the proof, since the theorem follows from Lemma 1.2 by standard methods of harmonic analysis (cf. [16, Chapter XVII, Theorems (1.20), (1.23)]).

2. Admissible convergence. We now cite, for the case of the maximal boundary $K / M$ only, Korányi's definition of unrestricted admissible convergence. (In this case unrestricted and semirestricted admissible convergence as defined in [13] coincide.)

For $T \in a$ and $C$ a compact subset of $X$, a truncated unrestricted admis. sible domain at the boundary point $k M$ is a set of the form

$$
\mathfrak{Q}_{C}^{T}(k M)=\left\{k a \cdot x \mid \log a-T \in \bar{a}_{+}, x \in C\right\} .
$$

A function $F$ on $X$ is said to converge admissibly and unrestrictedly to a value $L$ at $k M$ if, for every $C$ compact in $X$ and $\epsilon>0$, there exists a $T$ (depending on $C$ and $\epsilon$ ) such that, for all $x \in \mathbb{A}_{C}^{T}(k M),|F(x)-L|<\epsilon$.

It is clear that unrestricted admissible convergence at $k M$ does not depend on the choice of $k$ in the coset $k M$. A group-invariance property for unrestricted admissible convergence is proven in [12, Proposition 4.1].

Given a function $f$ on $K / M$ we may define a function $f_{1}$ on $\bar{N}$ by $f_{1}(\bar{n})=$ $f(k(\bar{n}) M)$. We say that $\bar{n}_{0}$ is a Lebesgue point of $f_{1}$ if

$$
\lim _{a \rightarrow+\infty} \frac{1}{\operatorname{mes}\left(V^{a}\right)} \int_{V^{a}}\left|f_{1}\left(\bar{n}_{0} \bar{n}\right)-f_{1}\left(\bar{n}_{0}\right)\right| d \bar{n}=0
$$


for some (hence any) compact neighborhood $V$ of $e$ in $\bar{N}$. According to $[13, \S 3$, Corollary 2], if $f_{1} \in L^{p}(\bar{N}), 1<p \leq \infty$, then almost all points of $\bar{N}$ are Lebesgue points of $f_{1}$. Since $k(\bar{N}) M$ is of full measure in $K / M$, and since the property of being a Lebesgue point is a local property, if $f \in L^{p}(K / M), 1<p \leq \infty$, almost all points of $K / M$ are of the form $k(\bar{n}) M$ with $\bar{n}$ a Lebesgue point of $f_{1}$. We shall call such points Lebesgue points of $f$. For $f \in L^{\infty}(K / M)$ we shall prove a Fatoutype theorem at Lebesgue points of $f$.

First, however, we prove a lemma which will be useful throughout this paper to extend results from radial or orispherical radial convergence to admissible convergence. This lemma appears to simplify all proofs of admissible convergence, even for the harmonic case (e.g., the results of [12], [13], and [14]), and to give some insight into the reason for considering this type of convergence.

2.1 Lemma. Let $C$ be a compact subset of $X, \lambda \in a^{*}-i a_{+}^{*}$ or rank $X=1$, $\lambda=0$. Then there exists a constant $C_{\lambda, C}$ (depending on $\lambda$ and $C$ alone) such that for all $k \in K, x \in C$,

$$
\left|P_{\lambda}(g \cdot x, k M)\right| \leq C_{\lambda, C}\left|P_{\lambda}(g \cdot o, k M)\right|
$$

whenever $g \in G$ is of the form $g=k_{1} a k_{2} ; k_{1}, k_{2} \in K ; \log a-T \in a_{+}$, for some $T \in a$ (depending on $\lambda$ and $C$ ).

Proof.

$$
\begin{aligned}
& \left|P_{\lambda}(g \cdot x, k M)\right|\left|P_{\lambda}(g \cdot o, k M)\right|^{-1} \\
& =\left(\left|\phi_{\lambda}(g \cdot o)\right|\left|\phi_{\lambda}(g \cdot x)\right|^{-1}\right)\left|e^{-(i \lambda+\rho)\left(H\left(g^{\prime-1} k\right)-H\left(g^{-1} k\right)\right)}\right|,
\end{aligned}
$$

where $g^{\prime}$ is such that $g^{\prime} \cdot o=g \cdot x$. We proceed to estimate each factor of the right-hand side of (2.1).

$$
\begin{aligned}
& \text { If } g=k_{1} a k_{2}, k_{2} \cdot x=\bar{n}_{1} a_{1} \cdot o, \\
& \qquad \begin{array}{l}
\left|\phi_{\lambda}(g \cdot o)\right|\left|\phi_{\lambda}(g \cdot x)\right|^{-1}=\left|\phi_{\lambda}(a \cdot o)\right|\left|\phi_{\lambda}\left(a \bar{n}_{1} a_{1} \cdot o\right)\right|^{-1} \\
=\left|\phi_{\lambda}(a \cdot o)\right|\left|\phi_{\lambda}\left(\bar{n}_{1} a a_{1} \cdot o\right)\right|^{-1}=\left|\phi_{\lambda}(a \cdot o)\right|\left|\phi_{\lambda}\left(a^{\prime} \cdot o\right)\right|^{-1},
\end{array}
\end{aligned}
$$

where $a^{\prime} \epsilon \exp \bar{a}_{+}$is such that

$$
\bar{n}_{1}^{a} a a_{1} \cdot o \in K a^{\prime} \cdot o \text {. }
$$

By Lemma 50 of [5] and the remark on uniformity in the proof of Lemma 1.1, since $\bar{n}_{1}$ and $a_{1}$ are confined to compact sets when $x$ is, and by (1.1) or (1.3) as appropriate, $\left|\phi_{\lambda}(g \cdot o)\right|\left|\phi_{\lambda}(g \cdot x)\right|^{-1}$ is bounded, for $\log a-T \epsilon a_{+}$for suitable $T \in a_{+}$, by any constant exceeding

$$
\left\{\left(\bar{n}_{1}, a_{1}\right) \mid \bar{n}_{2} a_{1} \cdot o \in K \cdot C \text { for some } \bar{n}_{2} \in \bar{n}_{1}^{\exp a_{+}}\left|e^{(i \lambda-\rho)\left(H\left(\bar{n}_{1}\right)+\log a_{1}\right)}\right| .\right.
$$


Now, $g^{\prime-1} g$ belongs to the compact set $D=\{g \in G \mid \circ \in g \cdot C\}$.

$$
H\left(g^{\prime-1} k\right)-H\left(g^{-1} k\right)=H\left(g^{\prime-1} g k\left(g^{-1} k\right)\right) \in H(D K) .
$$

Thus $\left|e^{-(i \lambda+\rho)\left(H\left(g^{\prime}-1 k\right)-H\left(g^{-1} k\right)\right)}\right|$ is bounded by $\operatorname{Max}_{b \in D K}\left|e^{-(i \lambda+\rho)(H(b))}\right|$.

We are now prepared to prove the principal result of this section.

2.2 Theorem. (i) Let $f \in L^{\infty}(K / M), k_{0} M$ be a Lebesgue point of $f, \lambda \in a^{*}$ $i a_{+}^{*}$. Then $F_{\lambda}$ converges admissibly and unrestrictedly to $f$ at $k_{0} M_{\text {. }}$ (In particular, $F_{\lambda}$ converges admissibly and unrestrictedly to $f$ at almost all points of $K / M$.

(ii) If rank $X=1$ this result bolds also for $\lambda=0$ and bolds for all finite regular signed measures on $K / M$. (By a Lebesgue point of a measure $\nu$, we mean a point $k\left(\bar{n}_{0}\right) M$ for which

$$
\lim _{a \rightarrow+\infty} \frac{1}{\operatorname{mes}\left(V^{a}\right)} \int_{\bar{n}_{0} V^{a}}|d \nu(k(\bar{n}))-L d \bar{n}|=0
$$

for some $L$; and in that case the convergence is to the limit L.)

Proof. According to [12, Proposition 4.2], $F_{\lambda}$ converges admissibly and unrestrictedly to $f\left(k\left(\bar{n}_{0}\right) M\right)$ at $k\left(\bar{n}_{0}\right) M$ if (and, in fact, only if) for every $\epsilon>0$ and every compact subset $U$ of $\bar{N}$ there exists a $T \epsilon a$ (depending on $\epsilon$ and $U$ ) such that $\left|F_{\lambda}(x)-f\left(k\left(\bar{n}_{0}\right) M\right)\right|<\epsilon$ whenever

$$
x \in \Gamma_{U}^{T}\left(\bar{n}_{0}\right)=\left\{x \in \bar{n}_{0} a U \cdot o \mid a \in A, \log a-T \in \bar{a}_{+}\right\} .
$$

For $x \in \bar{n}_{0} a U \cdot o, \log a-T \in \bar{a}_{+}$for a suitable $T$, we have, by Lemma 2.1,

$$
\begin{aligned}
\mid F_{\lambda}(x) & -f\left(k\left(\bar{n}_{0}\right) M\right)\left|\leq \int_{K / M}\right| f(k M)-f\left(k\left(\bar{n}_{0}\right) M\right)|| P_{\lambda}(x, k M) \mid d k_{M} \\
& \leq C_{\lambda, U \cdot o} \int_{K / M}\left|f(k M)-f\left(k\left(\bar{n}_{0}\right) M\right)\right|\left|P_{\lambda}\left(\bar{n}_{0} a \cdot o, k M\right)\right| d k_{M} \\
& =C_{\lambda, U \cdot o}\left|\phi_{\lambda}\left(\bar{n}_{0} a \cdot o\right)\right|^{-1} \int_{K / M}\left|f(k M)-f\left(k\left(\bar{n}_{0}\right) M\right)\right| \mid e^{-(i \lambda+\rho)\left(H\left(a^{-1} \bar{n}_{0}^{-1} k\right)\right) \mid d k_{M} .}
\end{aligned}
$$

By transferring the integration to $\bar{N}$ and applying the properties of the $H$-function as in the proof of Lemma 1.2, we find, after changing the variable $\bar{n}$ to $\bar{n}{ }_{0} \bar{n}$, that this is equal to

$$
\begin{aligned}
& C_{\lambda, U \cdot o}\left|e^{\left(\rho_{+} \lambda \lambda\right)(\log a)}\right|\left|\phi_{\lambda}\left(\bar{n}_{0} a \cdot o\right)\right|^{-1} \\
& \quad \cdot \int_{\bar{N}}\left|f_{1}\left(\bar{n}_{0} \bar{n}\right)-f_{1}\left(\bar{n}_{0}\right)\right|\left|e^{(i \lambda-\rho)\left(H\left(\bar{n}_{0} k(\bar{n})\right)\right)}\right|\left|e^{-(i \lambda+\rho)\left(H\left(\bar{n}^{-a^{-1}}\right)\right)}\right|\left|e^{(i \lambda-\rho)(H(\bar{n}))}\right| d \bar{n} .
\end{aligned}
$$

To prove (i) we note that, for $X \in a^{*}-i a_{+}^{*}$, by (1.1) and Lemma 50 of [5] (mentioned in the proof of Lemma 1.1), this is bounded, for a suitable choice of $T$, by 


$$
M_{\lambda, U} e^{2 \rho(\log a)} \int_{\bar{N}}\left|f_{1}\left(\bar{n}_{0} \bar{n}\right)-f_{1}\left(\bar{n}_{0}\right)\right|\left|e^{-(i \lambda+\rho)\left(H\left(\bar{n}^{-a^{-1}}\right)\right)}\right|\left|e^{(i \lambda-\rho)(H(\bar{n}))}\right| \mid d \bar{n},
$$

where $M_{\lambda, U}$ is any constant exceeding

$$
C_{\lambda, U \cdot o}|c(\lambda)|^{-1}\left|e^{(\rho-i \lambda)\left(H\left(\bar{n}_{0}\right)\right)}\right| \operatorname{Max}_{g \in \bar{n}_{0} K}\left|e^{(i \lambda-\rho)(H(g))}\right| .
$$

As in the proof of Lemma 1.2 (iv),

$$
\begin{aligned}
& \left|F_{\lambda}(x)-f\left(k\left(\bar{n}_{0}\right) M\right)\right| \\
& \quad \leq M_{\lambda, U} e^{2 \rho(\log a)} \int_{\bar{N}}\left|f_{1}\left(\bar{n}_{0} \bar{n}\right)-f_{1}\left(\bar{n}_{0}\right)\right| e^{-(\rho+\delta \eta)\left(H\left(\bar{n}^{-1}\right)\right) d \bar{n} .}
\end{aligned}
$$

We decompose the integral in (2.2) into an integral over $V^{a}$ and an integral over the rest of $\bar{N}$, where $V$ is a compact neighborhood of $e$ in $\bar{N}$.

$$
\begin{gathered}
e^{2 \rho(\log a)} \int_{\bar{N}-V^{a}}\left|f_{1}\left(\bar{n}_{0} \bar{n}\right)-f_{1}\left(\bar{n}_{0}\right)\right| e^{-(\rho+\delta \eta)\left(H\left(\bar{n}^{-1}\right)\right)} d \bar{n} \\
\leq 2\|f\|_{\infty} \int_{\bar{N}-V} e^{-(\rho+\delta \eta)(H(\bar{n}))} d \bar{n}<\frac{\epsilon}{2 M_{\lambda, U}}
\end{gathered}
$$

for a suitable choice of $V$ because the integral converges.

But

$$
\begin{aligned}
e^{2 \rho(\log a)} \int_{V^{a}}\left|f_{1}\left(\bar{n}_{0} \bar{n}\right)-f_{1}\left(\bar{n}_{0}\right)\right| e^{-(\rho+\delta \eta)\left(H\left(\bar{n}^{-a^{-1}}\right)\right)} d \bar{n} \\
\leq e^{2 \rho(\log a)} \int_{V^{a}}\left|f_{1}\left(\bar{n}_{0} \bar{n}\right)-f_{1}\left(\bar{n}_{0}\right)\right| d \bar{n} \\
\quad=\frac{\operatorname{mes}(V)}{\operatorname{mes}\left(V^{a}\right)} \int_{V^{a}}\left|f_{1}\left(\bar{n}_{0} \bar{n}\right)-f_{1}\left(\bar{n}_{0}\right)\right| d \bar{n}<\frac{\epsilon}{2 M_{\lambda, U}}
\end{aligned}
$$

for a suitable choice of $T$, by the definition of Lebesgue point. Therefore

$$
\left|F_{\lambda}(x)-f\left(k\left(n_{0}\right) M\right)\right|<\epsilon .
$$

To prove (ii), we let rank $X=1, V$ be a compact neighborhood of $e$ in $\vec{N}$, and, for suitable $a_{0} \in A$, we estimate the integral

$$
\begin{aligned}
& \int_{k\left(\bar{n}_{0} V^{\left.a_{0}\right) M}\right.}\left|f(k M) d k_{M}-L d k_{M}\right|\left|P_{\lambda}(x, k M)\right| \\
& =\int_{V^{a_{0}}}\left|f\left(\bar{n}_{0} \bar{n}\right) d \bar{n}-L d \bar{n}\right|\left|P_{\lambda}\left(x, k\left(\bar{n}_{0} \bar{n}\right) M\right)\right| e^{-2 \rho\left(H\left(\bar{n}_{0} \bar{n}^{n}\right)\right)}
\end{aligned}
$$

as in the proof of (i), using the fact that $\bar{n}_{0}$ is a Lebesgue point of $f_{1}$, where $L$ is as in the definition of Lebesgue point. (For $f$ a function, $L=f\left(k\left(n_{0}\right) M\right)$ a.e.) (In this case, since $\operatorname{dim} a=1$, an easy generalization of the classical HardyLittlewood maximal theorem (cf. [16, Chapter XVII, (3.5) and (2.1)]) yields that 
almost all points are Lebesgue points even for $L^{1}$ functions and for finite regular signed ineasures.)

We estimate the integral over the rest of $K / M$ as follows:

$$
\int_{K / M-k\left(\bar{n}_{0} V\right.} a_{0}\left|f(k M) d k_{M}-L d k_{M}\right|\left|P_{\lambda}(x, k M)\right|
$$

$$
\leq C_{\lambda, U \cdot \circ} \int_{K / M-k\left(\bar{n}_{0} V\right.} i^{a_{0}}{ }_{M}\left|f(k M) d k_{M}-L d k_{M}\right|\left|P_{\lambda}\left(\bar{n}_{0} a \cdot o, k M\right)\right| .
$$

By a suitable choice of $T$ we can make $\bar{n}_{0} a \cdot o=k^{\prime} a^{\prime}$. o with $k^{\prime} \in W$, where $\left.\bar{W} \subset k\left(\bar{n}_{0} V^{a}\right)\right) M$. Then the expression (2.3) is bounded by

$$
\begin{aligned}
& C_{\lambda, U \cdot \circ}\left\{\sup _{\log a^{\prime}-T \in a_{+} ; l \in W^{-1}\left(K / M-k\left(\bar{n}_{0} V^{a^{0}}{ }^{M}\right)\right.}\left|P_{\lambda}\left(a^{\prime} \cdot o, l M\right)\right|\right\} \\
& \cdot\left(L+\left\|f(k M) d k_{M}\right\|_{\nu}\right),
\end{aligned}
$$

where \|\|$_{\nu}$ denotes the variation norm. But, for $\lambda=0$ by (1.6), and for $\lambda=$ $(u-i v) \alpha, v>0$, by a similar application of (1.4) and (1.5) which yields

$$
P_{\lambda}(a \cdot o, k(\bar{n}) M)
$$

$$
\sim C_{\lambda} e^{2 \rho(\log a)}\left|\frac{\left(1+r|X|^{2}\right)^{2}+4 r|Y|^{2}}{\left(1+r e^{2 a(\log a)}|X|^{2}\right)^{2}+4 r e^{4 a(\log a)}|Y|^{2}}\right|^{1 / 4\left(m_{a}+2 m_{2 a}+2 i[u-i v]\right)},
$$

where notations are as in (1.5), we see that the supremum in (2.4) may be made as small as desired.

While the local result of Theorem 2.2(i) for Lebesgue points cannot be generalized for arbitrary-rank spaces to $L^{p}(K / M)$ for any finite $p$ (cf. [16, at the top of p. 305]), there is some hope for a global result asserting unrestricted admissible convergence on some unspecified set of full measure. A result in this direction, but with severe restrictions on both $\lambda$ and $p$, follows. A more complete result for products of rank-one spaces will be shown in the next section.

2.3 Theorem. Let $H_{0}$ be the element of $a_{+}$for which $\beta\left(H_{0}\right)=1$ for every simple restricted root $\beta, p_{0}=2 \rho\left(H_{0}\right)-1, f \in L^{p}(K / M) \cap L^{1}(K / M)$ for some $p>p_{0}$. Let $\lambda \in-i \rho+a^{*}-i \bar{a}{ }_{+}^{*}$. Then $F_{\lambda}$ converges admissibly and unrestrict edly to $f$ almost everywhere. If $X=\operatorname{SL}(n, \mathbf{R}) / \mathrm{SO}(n)$, this result bolds for $p>$ $\left[n^{2} / 4\right]-1$. (By a simple restricted root we mean a positive eigenvalue of $\operatorname{ad}_{\mathfrak{g}}(a)$ which is not the sum of two positive eigenvalues of $\operatorname{ad}_{\mathbf{g}}(a)$.)

Proof. The theorem for the harmonic case $(\lambda=-i \rho)$ is proven by Lindahl [14], using maximal function estimates. From (1.4) we have, for $\lambda \epsilon a^{*}-i a_{+}^{*}$, 


$$
P_{\lambda}(a \cdot o, k(\bar{n}) M) \sim C_{\lambda} e^{2 \rho(\log a)} e^{-(i \lambda+\rho)\left(H\left(\bar{n} a^{-1}\right)-H(\bar{n})\right)} .
$$

Since $H\left(\bar{n}^{a^{-1}}\right)-H(\bar{n})=H\left(a^{-1} k(\bar{n})\right)+\log a \epsilon\left(a_{+}^{*}\right) *$ for $a \in \exp a_{+}$by Lemma 35 of [4], it follows immediately that, for $\lambda_{0} \in a^{*}-i a_{+}^{*}, \lambda \in \lambda_{0}+a^{*}-i \bar{a}_{+}^{*}$, as $a \rightarrow+\infty$ we have eventually

$$
\left|P_{\lambda}(a \cdot o, k(\bar{n}) M)\right| \leq C_{\lambda_{0}, \lambda}\left|P_{\lambda_{0}}(a \cdot o, k(\bar{n}) M)\right|,
$$

where $C_{\lambda_{0}, \lambda}$ is any constant exceeding $\left|C_{\lambda}\right|\left|C_{\lambda_{0}}\right|^{-1}$. Setting $\lambda_{0}=-i \rho$-and, of course, using Theorem 1.3(i) in place of the corresponding theorem for $\lambda=-i \rho-$ we can now extend the proof of Lindahl to our more general case.

3. Products of spaces of rank one. In the case of a symmetric space of rank one, the asymptotic behavior of the kernel $P_{\lambda}$ is known quite explicitly for $\lambda \in a^{*}-i a_{+}^{*}(2.5)$ and for $\lambda=0$ (1.6). Also available in the rank-one case but not in general is a maximal theorem of Knapp [10, Theorem 5.1], which we shall state shortly after introducing some required notation.

For $\bar{n} \in \bar{N}$ we set $|\bar{n}|=\operatorname{Max}\left\{|X|,|Y|^{1 / 2}\right\}$, where $X, Y,||$, are as in (1.5). We set $B(R)=\{\bar{n} \in \bar{N}|| \bar{n} \mid \leq R\}$. For $k_{0} M \in K / M$ we set $B_{R}\left(k_{0} M\right)=k_{0} k(B(R)) M$, $0<R<\infty ; B_{\infty}\left(k_{0} M\right)=K / M$. One verifies easily that this definition does not depend upon the representative $k_{0}$ of the coset $k_{0} M$.

For $f \in L^{1}(K / M)$ we define the maximal function

$$
f^{*}\left(k_{0} M\right)=\sup _{0<R \leq \infty} \frac{1}{\operatorname{mes}\left(B_{R}\left(k_{0} M\right)\right)} \int_{B_{R}\left(k_{0} M\right)}|f(k M)| d k_{M^{*}}
$$

The following lemma is due to Knapp [10, Theorem 5.1].

3.1 Lemma. For $f \in L^{1}(K / M), \xi>0$,

$$
\operatorname{mes}\left(\left\{k M \mid f^{*}(k M) \geq \xi\right\}\right) \leq C^{\prime} \xi^{-1}\|f\|_{1},
$$

where $C^{\prime}$ is a constant (independent of $f, \xi$ ).

Knapp's lemma has the following corollary, which can be proven by a standard interpolation method (as in [15, Chapter 1, Theorem (13.15)(i)]).

3.2 Corollary. If $f \in L^{p}(K / M), 1<p \leq \infty$, then

$$
f^{*} \in L^{p}(K / M) \text { and }\left\|f^{*}\right\|_{p} \leq A_{p}\|f\|_{p} \text {, }
$$

where $A_{p}$ is a constant (independent of $f$ ).

We now use Knapp's maximal function $f^{*}$ to estimate the generalized Poisson integrals $F_{\lambda}$ of $f$, as follows: 
3.3 Lemma. Let rank $X=1, \lambda=0$ or $\lambda \epsilon a^{*}-i a_{+}^{*}, f \in L^{1}(K / M), C$ be compact in $X$. Then for a suitable choice of $T$ (depending on $\lambda$ and $C$ but not on $k$ and $f$ ) there exists a constant $A_{\lambda, C}$ (independent of $k, f$ ) sucb that $\left|F_{\lambda}(x)\right| \leq A_{\lambda, C^{f}} *(k M)$ for all $x \in \mathbb{Q}_{C}^{T}(k M)$.

Proof. Since the operator $*$ commutes with translations by $K$, it suffices to prove the lemma for $k=e$. We write $B_{R}$ for $B_{R}(e M)$.

For $x \in \mathbb{A}_{C}^{T}(e M), x=a \cdot y, y \in C, \log a-T \in \bar{a}_{+}$. For a suitable choice of $T$ we have, by Lemma 2.1,

$$
\begin{aligned}
\left|F_{\lambda}(x)\right| & \leq \int_{K / M}|f(k M)|\left|P_{\lambda}(a \cdot y, k M)\right| d k_{M} \\
& \leq C_{\lambda, C} \int_{K / M}|f(k M)|\left|P_{\lambda}(a \cdot o, k M)\right| d k_{M} .
\end{aligned}
$$

To simplify notation we shall set $t=\alpha(\log a)$. (Hence $a=\exp t H_{0}, H_{0}$ as in Theorem 2.3.) We shall also set $\lambda=(u-i v) \alpha, u=v=0$ or $v>0$. From (2.5) it follows immediately that, for sufficiently large $t$, for $v>0$, for some constant $A_{\lambda}$,

$$
\left|P_{\lambda}\left(\exp t H_{0} \cdot o, k(\bar{n}) M\right)\right| \leq\left\{\begin{array}{l}
A_{\lambda} e^{\left(m_{a}+2 m_{2 \alpha}\right) t}, \quad \bar{n} \text { arbitrary, } \\
A_{\lambda} e^{-2 v t}|\bar{n}|^{-\left(m_{a}+2 m_{2 a}+2 v\right)}, \quad|\bar{n}| \leq 1, \\
A_{\lambda} e^{-2 v t}, \quad|\bar{n}|>1 .
\end{array}\right.
$$

From (1.6) it follows that, for sufficiently large $t$, for some constant $A_{0}$,

$$
\left|P_{0}\left(\exp t H_{0} \cdot o, k(\bar{n}) M\right)\right| \leq \begin{cases}A_{0} t^{-1} e^{\left(m_{\alpha}+2 m_{2 \alpha}\right) t}, & \bar{n} \text { arbitrary } \\ A_{0} t^{-1}|\bar{n}|^{-\left(m_{a}+2 m_{2 \alpha}\right)}, & |\bar{n}| \leq 1 \\ A_{0} t^{-1}, \quad|\bar{n}|>1 . & \end{cases}
$$

In the sequel we shall always assume $t$ large enough for estimate (3.2) or (3.3) to hold and $t \geq 1$.

We can now break up the integral on the right-hand side of (3.1) into three integrals-over the sets $B_{e^{-t}}, B_{1}-B_{e^{-t}}$, and $B_{\infty}-B_{1}$.

We estimate the integral over $B_{e^{-t}}$ by the first inequality in (3.2) or (3.3). 
$\int_{B}|f(k M)|\left|P_{\lambda}\left(\exp t H_{0} \cdot o, k M\right)\right| d k_{M}$

$$
\begin{aligned}
& \leq A_{\lambda} e^{\left(m_{a}+2 m_{2 a}\right) t} \int_{B}|f(k M)| d k_{M} \\
& =A_{\lambda^{-t}} \operatorname{mes}(B(1))\left[\operatorname{mes}\left(B\left(e^{-t}\right)\right)\right]^{-1} \int_{B}|f(k M)| d k_{M} \\
& \leq A_{\lambda^{-t}} \operatorname{mes}(B(1))\left[\operatorname{mes}\left(B_{e^{-r}}\right)\right]^{-1} \int_{B}|f(k M)| d k_{M} \leq A_{\lambda^{-t}} \operatorname{mes}(B(1)) f *(e M) .
\end{aligned}
$$

Next we estimate the integral over $B_{\infty}-B_{1}$ by the last inequality in (3.2) or (3.3):

$$
\begin{aligned}
\int_{B-B_{1}}|f(k M)|\left|P_{\lambda}\left(\exp t H_{0} \cdot o, k M\right)\right| d k_{M} & \leq A_{\lambda} e^{-2 v} \int_{B_{\infty}}|f(k M)| d k_{M} \\
& \leq A_{\lambda} e^{-2 v} f *(e M) .
\end{aligned}
$$

To estimate the integral over $B_{1}-B_{e^{-t}}$ by the second inequality in (3.2) or (3.3), we must consider separately the cases $v>0$ and $u=v=0$ and must further subdivide, as follows.

For $v>0$,

$$
\begin{aligned}
\int_{B_{1}-B} e^{-t} & |f(k M)|\left|P_{\lambda}\left(\exp t H_{0} \cdot o, k M\right)\right| d k_{M} \\
& =\sum_{j=0}^{\infty} \int_{B_{1} B_{2} j+1} e^{-t-B} 2^{j} e^{-t}|f(k M)|\left|P_{\lambda}\left(\exp t H_{0} \cdot o, k M\right)\right| d k_{M} \\
& \leq \sum_{j=0}^{\infty} A_{\lambda} e^{\left(m_{a}+2 m_{2 a}\right) t} 2^{-\left(m_{a}+2 m_{2 a}+2 v\right) j} \int_{B_{2^{j+1}} e^{-t}}|f(k M)| d k_{M}
\end{aligned}
$$

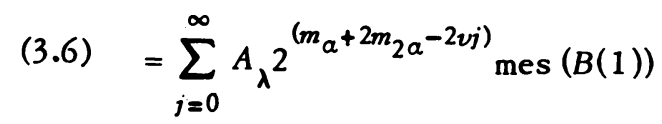

$$
\text { . }\left[\operatorname{mes}\left(B\left(2^{j+1} e^{-t}\right)\right)\right]^{-1} \int_{B, j+1} e^{-t}|f(k M)| d k_{M}
$$

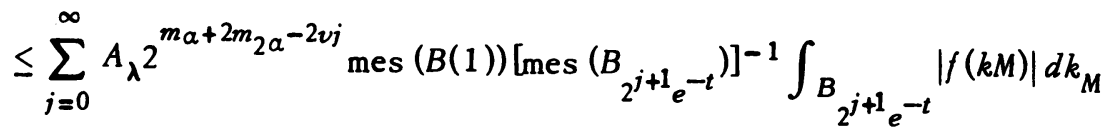

$$
\begin{aligned}
& \leq \sum_{j=0}^{\infty} A_{\lambda} 2^{m_{a}+2 m_{2 a^{-2 v j}}} \operatorname{mes}(B(1)) f *\left(e^{\prime} M\right)=A \frac{2^{m_{a}+2 m} 2 a}{1-2^{-2 v}} \operatorname{mes}(B(1)) f *(e M) .
\end{aligned}
$$




$$
\begin{aligned}
& \int_{B_{1}-B_{e^{-t}}}|f(k M)|\left|P_{0}\left(\exp t H_{0} \cdot o, k M\right)\right| d k_{M} \\
& =\sum_{j=0}^{\infty} \int_{B_{1} \cap B_{2}{ }^{j+1} e^{-t}{ }^{-B}{ }_{2^{j} e^{-t}}}|f(k M)|\left|P_{0}\left(\exp t H_{0} \cdot o, k M\right)\right| d k_{M} \\
& \leq \sum_{j=0}^{[t / \log 2]_{+1}} A_{0} t^{-1} e^{\left(m_{a}+2 m_{2 a}\right) t} 2^{-\left(m_{a}+2 m_{2 a}\right) j} \int_{B_{2}{ }^{j+1} e^{-t}}|f(k M)| d k_{M} \\
& =\sum_{j=0}^{[/ / \log 2]+1} A_{0} t^{-12^{m} a+2 m} 2 a_{\operatorname{mes}}(B(1)) \\
& \cdot\left[\operatorname{mes}\left(B\left(2^{j+1} e^{-t}\right)\right)\right]^{-1} \int_{B}{ }_{2^{j+1} e^{-t}}|f(k M)| d k_{M}
\end{aligned}
$$

$$
\begin{aligned}
& \leq \sum_{j=0}^{[t / \log 2]+1} A_{0} t^{-1} 2^{m_{a}+2 m_{2 \alpha}} \operatorname{mes}(B(1)) \\
& \text {. }\left[\operatorname{mes}\left(B_{2^{j+1} e^{-t}}\right)\right]^{-1} \int_{B}{ }_{2^{j+1} e^{-t}}|f(k M)| d k_{M} \\
& \leq\{[t / \log 2]+1\} A_{0} t^{-1} 2^{m a+2 m} 2 a \operatorname{mes}(B(1)) f *(e M) \\
& \leq\{1 / \log 2+1\} A_{0} 2^{m_{a}+2 m_{2} a_{\text {mes }}(B(1)) f^{*}(e M) .}
\end{aligned}
$$

By combination of the estimates in (3.1), (3.4), (3.5), and (3.6) or (3.7), the lemma follows.

The preceding lemmas enable us to prove the principal result of this section.

3.4 Theorem. Let $X=\Pi_{i=1}^{m} X_{i}$, rank $X_{i}=1$. Let $f \in L^{p}(K / M), 1<p \leq \infty$. Let $\lambda=\sum_{i=1}^{m}\left(u_{i}-i v_{i}\right) \alpha_{i}$, where $\alpha_{i}$ is the (smaller) positive eigenvalue of $\operatorname{ad}_{p}\left(a_{i}\right)$, where $a_{i}$ is the Cartan subspace of the space $X_{i}, u_{i}=v_{i}=0$ or $v_{i}>0$. Then $F_{\lambda}$ converges admissibly and unrestrictedly to $f$ almost everywbere.

Proof. Since $L^{\infty}(K / M)$ is contained in every $L^{p}(K / M)$, we may assume $p<\infty$. Then we may fix $\epsilon>0$ and set $f=f^{1}+f^{2}, f^{1} \in C(K / M),\left\|f^{2}\right\|_{p}<\epsilon^{1+1 / p}$.

Fix $C$ compact in $X$, and let $x_{k M} \in \mathbb{A}_{C}^{T}(k M)$.

For suitable $T$, independent of $k M,\left|F_{\lambda}^{1}\left(x_{k M}\right)-f^{-1}(k M)\right|<\epsilon$ by Theorem 1.3(i) and Lemma 1.1.

$$
\left\{k M|| F_{\lambda}^{2}\left(x_{k M}\right)-f^{2}(k M) \mid \geq 2 \epsilon\right\} \subset\left\{k M|| f^{2}(k M) \mid \geq \epsilon\right\} \cup\left\{k M|| F_{\lambda}^{2}\left(x_{k M}\right) \mid \geq \epsilon\right\} .
$$




$$
\operatorname{mes}\left(\left\{k M|| f^{2}(k M) \mid \geq \epsilon\right\}\right)<\epsilon .
$$

Since we may assume that $C$ is a product $C=\Pi_{i=1}^{m} C_{i}, C_{i}$ compact in $X_{i}$; by iteration of Lemma 3.3, applied to each $X_{i}$, for suitable choice of $T$,

$$
\left\|F_{\lambda}^{2}\left(x_{k M}\right)\right\|_{L}^{p}(K / M) \leq A_{\lambda, C} f_{1 \cdots m}^{2 *}(k M)
$$

for some constant $A_{\lambda, C}$, where ${ }_{i}^{*}$ indicates the Knapp maximal operator of the space $X_{i}$ acting on $f$ regarded as a function on $K_{i} / M_{i}$, the maximal boundary of $X_{i}$, other coordinates of $k M$ being held fixed. By iteration of Lemma 3.2, for some constant $A_{p}$,

so that

$$
\left\|f_{1 \cdots m}^{2 *}\right\|_{p}^{*} \leq A_{p}\left\|f^{2}\right\|_{p}
$$

$$
\begin{gathered}
\left\|F_{\lambda}^{2}\left(x_{k M}\right)\right\|_{L^{p}(K / M)} \leq A_{\lambda,} C^{A}{ }_{p}\left\|f^{2}\right\|_{p}<A_{\lambda}, C^{A} \epsilon^{1+1 / p} . \\
\operatorname{mes}\left(\left\{k M|| F_{\lambda}^{2}\left(x_{k M}\right) \mid \geq \epsilon\right\}\right)<\left(A_{\lambda}, C^{A}{ }_{p}\right)^{p} \epsilon .
\end{gathered}
$$

Therefore $\left|F_{\lambda}\left(x_{k M}\right)-f(k M)\right|<3 \epsilon$ except on a set of measure less than $\left(1+\left(A_{\lambda, C^{A}}{ }^{p}\right)^{p}\right) \epsilon$.

Since $\epsilon$ is arbitrary and $x_{k M}$ is arbitrary in $\mathbb{Q}_{C}^{T}(k M)$, the theorem follows.

In the case $v_{i}>0$ the result of Theorem 3.4 cannot be extended to $f \in L^{1}(K / M)$. A counterexample can be constructed by the method of [16, Chapter XVII, Theorem (2.14)(ii)]. For a more restricted type of convergence, however, a positive result will be stated below (Theorem 3.5).

In the case $u_{i}=v_{i}=0$ for some $i$, the boundary behavior of $F_{\lambda}$ for $f \in L^{1}(K / M)$ remains an open question.

We now give some definitions meaningful in any symmetric space of noncompact type. These definitions in the ir present form are due to Korányi [1 2, \$2].

A truncated restricted admissible domain at a boundary point $k M$ with respect to an element $H \in a_{+}$is a set of the form

$$
\mathbb{R}_{H, C}^{\tau}(k M)=\{k \exp t H \cdot x \mid t \geq \tau, x \in C\}
$$

for some $\tau \in \mathbf{R}, C$ compact in $X$.

A function $F$ on $X$ converges admissibly and restrictedly with respect to $H$ to a number $L$ at $k M$ if for every $C$ compact in $X, \epsilon>0$, there exists a number $\tau$ such that $|F(x)-L|<\epsilon$ for all $x \in R_{H, C}^{\tau}(k M)$. One verifies easily that this definition does not depend upon the choice of the representative $k$ of the coset $k M$.

By iteration of the estimates (3.2), one can prove by standard methods of harmonic analysis the following theorem. 
3.5 Theorem. Let $X=\prod_{i=1}^{m} X_{i}$, rank $X_{i}=1, f$ be a finite regular signed measure on $K / M, \lambda \in a^{*}-i a_{+}^{*}, H \in a_{+}$. Then $F_{\lambda}$ converges admissibly and re. strictedly to $f$ with respect to $H$ almost everywbere.

Remark. Dr. Korányi has recently called to my attention a letter of Lindahl dated September 6, 1971, which proves Lemma 2.1 for the harmonic case and suggests the use of this lemma to extend results on orispherical radial convergence to admissible convergence. Although Lindahl's proof for this case has priority, mine is independent. The same letter of Lindahl also mentions results of Knapp and E. M. Stein improving the conditions on $p$ in Theorem 2.3 for certain symmetric spaces. In $\mathrm{SL}(n, \mathbf{R}) / \mathrm{SO}(n)$ the theorem holds for $p>n-2$, and in $\mathrm{S}_{\mathrm{p}}(2, \mathbf{R}) / U(2)$ it holds for $p>2$.

\section{REFERENCES}

1. A. Erdélyi et al., Higher transcendental functions. Vol. I. The hypergeometric function, Legendre functions, McGraw-Hill, New York, 1953. MR 15, 419.

2. H. Furstenberg, Translation-invariant cones of functions on semi-simple Lie groups, Bull. Amer. Math. Soc. 71 (1965), 271-326. MR 31 \#1326.

3. S. G. Gindikin and F. I. Karpelevič, Plancherel measure of Riemannian symmetric spaces of nonpositive curvature, Dokl. Akad. Nauk SSSR 145 (1962), 252-255 = Soviet Math. Dok1. 3 (1962), 962-965. MR 27 \#240.

4. Harish-Chandra, Spherical functions on a semisimple Lie group. I, Amer. J. Math. 80 (1958), 241-310. MR $20 \# 925$.

5. —_ Spherical functions on a semisimple Lie group. II, Amer. J. Math. 80 (1958), 553-613. MR $21 \# 92$.

6. S. Helgason, $A$ duality for symmetric spaces with applications to group representations, Advances in Math. 5 (1970), 1-154. MR $41 \# 8587$.

7. - Group representations and symmetric spaces, Proc. Internat. Congress Math. (Nice, 1970), Vol. 2, Gauthier-Villars, Paris, 1971, pp. 313-319.

8. S. Helgason and A. Korányi, A Fatou-type theorem for harmonic functions on symmetric spaces, Bull. Amer. Math. Soc. 74 (1968), 258-263. MR 37 \#4753.

9. F. I. Karpelevič, The geometry of geodesics and the eigenfunctions of the Beltrami-Laplace operator on symmetric spaces, Trudy Moskov. Mat. Obšć. 14 (1965), 48-185 = Trans. Moscow Math. Soc. 1965, 51-199. MR 37 \#6876.

10. A. W. Knapp, Fatou's theorem for symmetric spaces. I, Ann. of Math. (2) 88 (1968), 106-127. MR 37 \#1528.

11. A. W. Knapp and R. E. Williamson, Poisson integrals and semisimple Lie groups, J. Analyse Math. 24 (1971), 53-76.

12. A. Korányi, Boundary behavior of Poisson integrals on symmetric spaces, Trans. Amer. Math. Soc. 140 (1969), 393-409. MR 39 \#7132.

13. - Harmonic functions on symmetric spaces, Geometry and Harmonic Analysis of Symmetric Spaces, Dekker, New York (to appear).

14. L. - $\AA$. Lindahl, Fatou's theorem for symmetric spaces, Mittag-Leffler Institute, 1971. (manuscript) 
15. A. Zygmund, Trigonometrical series. Vol. I, 2nd rev. ed., Cambridge Univ. Press, New York, 1959. MR 21 \#6498.

16. __, Trigonometrical series. Vol. II, 2nd rev. ed., Cambridge Univ. Press, New Yqrk, 1959. MR 21 \#6498.

DEPARTMENT OF MATHEMATICS, MASSACHUSETTS INSTITUTE OF TECHNOLOGY, CAMBRIDGE, MASSACHUSETTS 02139 